\title{
Effect of Curcuma longa L. extract on noninvasive cardiovascular biomarkers in hypertension animal models
}

\author{
Patonah Hasimun* (D), Agus Sulaeman, Arif Hidayatullah, Yani Mulyani \\ Department of Pharmacology Research Group, Faculty of Pharmacy, Bhakti Kencana University, Bandung, Indonesia.
}

\begin{tabular}{|c|c|}
\hline ARTICLE INFO & ABSTRACT \\
\hline $\begin{array}{l}\text { Received on: } 27 / 12 / 2020 \\
\text { Accepted on: } 01 / 05 / 2021 \\
\text { Available online: } 05 / 08 / 2021\end{array}$ & $\begin{array}{l}\text { Arterial stiffness and QRS-T angle (the spatial angle between the vectors of the T-wave and QRS loops on typical } \\
\text { electrocardiogram) are essential biomarkers for estimating the risk of cardiovascular events in hypertensive patients. } \\
\text { Turmeric or Curcuma longa L., which belongs to the Zingiberaceae family, is reported to have antihypertensive } \\
\text { activity. However, its effect on these biomarkers is unknown. This research investigates the antihypertensive effect }\end{array}$ \\
\hline $\begin{array}{l}\text { Key words: } \\
\text { Curcuma longa L. } \\
\text { (Zingiberaceae), } \\
\text { hypertension, arterial } \\
\text { stiffness, QRS-T angle, nitric } \\
\text { oxide. }\end{array}$ & $\begin{array}{l}\text { of turmeric extract on arterial stiffness and frontal plane QRS-T angle in hypertensive animal models. High blood } \\
\text { pressure was induced by a high-fat and high-fructose (HFHF) diet for } 28 \text { days in male Wistar rats. A daily dose of } \\
\text { turmeric extract }(50,100 \text {, and } 200 \mathrm{mg} / \mathrm{kg} \text { ) or captopril was administered to hypertensive animals for } 14 \text { days. Blood } \\
\text { pressure, arterial stiffness, heart rate (HR), QRS-T angle, and nitric oxide (NO) levels were evaluated. An HFHF diet } \\
\text { triggers a decrease in NO serum levels resulting in significantly increased arterial stiffness, which correlates with } \\
\text { increased systolic blood pressure and diastolic blood pressure due to ventricular dysfunction supported by the wide } \\
\text { QRS-T angle, and also increased HR. Turmeric extract significantly enhances the bioavailability of NO vasodilators, } \\
\text { effectively reversing all the hypertensive-induced changes studied. This extract is helpful as a vasodilator that lowers } \\
\text { blood pressure by repairing arterial stiffness and preventing ventricular dysfunction of the heart. }\end{array}$ \\
\hline
\end{tabular}

\section{INTRODUCTION}

Increased aortic stiffness has emerged as a significant risk factor for target organ damage and cardiovascular disease events over the last decade. Aortic stiffness can be measured using pulse wave velocity (PWV), which is influenced by wall stiffness and the flow-diameter interaction. Stiffness has been shown in recent studies to predate and lead to the pathogenesis of hypertension (Mitchell, 2014). The left ventricle is filled by aortic stiffening, which increases early and late systolic load. These changes can cause ventricular remodeling and impair systolic and diastolic function. Moreover, the QRS and T-wave angles expand due to an imbalance in electrical activation and recovery (Selvaraj et al., 2014). Therefore, arterial stiffness and the QRS-T angle are essential biomarkers for cardiovascular risk prediction.

${ }^{*}$ Corresponding Author

Patonah Hasimun, Department of Pharmacology Research Group, Faculty of Pharmacy, Bhakti Kencana University, Bandung, Indonesia. E-mail:patonah@bku.ac.id
Recently reported, clinical noninvasive methods are beneficial in assessing cardiovascular diseases because they are easy to use and accurate in measuring the risks of the conditions. These methods were considered helpful to clinicians in providing therapy to patients without performing surgical procedures (Sun, 2015). Therefore, their practical application is encouraged, especially in revealing the effect and mechanism of drug action that can affect arterial stiffness (Stephane et al., 2012) and QRS-T angle (Oehler et al., 2014). Also, arterial stiffness and the QRS-T angle are essential biomarkers for assessing the success of antihypertensive drug therapy (Niiranen et al., 2016).

Animal models have been developed to study the effects of drugs that can affect cardiovascular diseases, including hypertension. Moreover, Sharma et al. (2007) showed that feeding animals could establish animal models of hypertension with an high-fat and high-fructose (HFHF) diet. According to Komnenov et al. (2019), an HFHF diet induces arterial stiffness. Physiologically, arterial stiffness continues to rise with ageing. In previous research, we developed a noninvasive method to measure arterial stiffness using the PWV method in mice aged 1 month and aged 3 months. There is a significant increase in arterial stiffness 
with ageing (Zakaria and Hasimun, 2017). Also, this method was designed to measure the frontal plane QRS-T axis angle (Zakaria and Hasimun, 2019).

Turmeric or Curcuma longa L. (Zingiberaceae) has been widely studied and reported to affect cardiovascular function, including hypertension. The preclinical and clinical studies showed its antihypertensive effect when used as a single extract (Akinyemi et al., 2016) and in combination with other herbs, including garlic (Sukandar et al., 2010). However, its effect on arterial stiffness and frontal plane QRS-T angle biomarkers has not been reported. Therefore, this study aimed to determine the impact of turmeric as an antihypertensive agent and its effect on arterial stiffness and QRS-T angle in animal models of hypertension induced by HFHF diet.

\section{MATERIALS AND METHODS}

\section{Turmeric collection and botanical authentication}

Turmeric was obtained from the Manoko, Lembang Plantation, Bandung, West Java, Indonesia, and the species was certified under the number 5948/I1.CO2.2/PL/2018 at the School of Biological Science and Technology Laboratory, Bandung Institute of Technology.

\section{Sources of chemicals and drugs}

Dimethyl sulfoxide, $70 \%$ ethanol, aqua pro injection, $\mathrm{ZnSO}_{4} 6 \%, \mathrm{CaCl} 6 \%$, acetic acid $1 \mathrm{~N}$, sulfanilic acid, $\mathrm{N}-(1-$ naphthyl ethyl diamine dihydroxide), and sodium nitric were provided by the department of integrated laboratory, Bhakti Kencana University. Captopril, as a reference drug, is a generic drug purchased from a local pharmacy.

\section{Preparation and extraction of turmeric}

The turmeric rhizome was cleaned to remove soil, foreign particles, and other plant parts before being washed under running water. The rhizome was then cut into small pieces and dried at a specific temperature in a $45^{\circ} \mathrm{C}$ oven. Dried samples were mashed and placed in an airtight jar.

Turmeric powder was extracted for 72 hours using $70 \%$ ethanol $(1: 10 \mathrm{~b} / \mathrm{v})$ by maceration. The supernatant was filtered and evaporated at a temperature of $50^{\circ} \mathrm{C}$ using a rotary vacuum evaporator. Each processed a total of $300 \mathrm{~g}$ of turmeric rhizome powder; then $30 \mathrm{~g}$ of the extract was obtained. Hence, the yield was $10 \%$ of the total.

\section{HFHF diet preparation}

$1,000 \mathrm{~g}$ of regular chow and $400 \mathrm{~g}$ of fat were combined to make a $40 \%$ fat feed. The fat contained $155 \mathrm{~g}$ of butter, $90 \mathrm{~g}$ of eggs ( 1 duck egg and four quail eggs), and $155 \mathrm{~g}$ of beef fat. High fructose was prepared by dissolving $25 \mathrm{~g}$ of fructose in $100 \mathrm{ml}$ of distilled water as drinking water.

\section{Animal handling and care}

Male Wistar rats, 2 months old, were obtained from the D-Wistar Laboratory in Bandung, Indonesia, for this research. The animals were housed in cages with normal conditions, such as a $25^{\circ} \mathrm{C}$ room temperature and relative humidity, a 12-hour lightdark period, and free access to standard food and drinking water for 7 days. This research protocol followed ethical requirements and was approved by the Ethics and Research Committee, Faculty of Medicine, Padjadjaran University, based on letter number 640/ UN6-KEP/EC/2019.

\section{Experimental design}

Male Wistar rats were randomly divided into six groups (six rats per group) as follows: groups 1 and 2, no treatment; group 3, treated with a daily oral dose of $2.5 \mathrm{mg} / \mathrm{kg}$ captopril; and groups 4-6, treated with a daily dose of 50,100 , and $200 \mathrm{mg} / \mathrm{kg}$ of turmeric extract, respectively. All groups received an HFHF diet for 28 days, except group 1 . On day 28 , blood pressure, heart rate (HR), PWV, frontal QRS-T angle, and serum levels of nitric oxide (NO) were then measured.

\section{Blood pressure measurement}

The systolic and diastolic blood pressure systolic blood pressure (SBP) and diastolic blood pressure (DBP) of conscious rats were assessed using the $\mathrm{CODA}^{\circledR}$ Mouse and Rat Tail-Cuff Blood Pressure System (KENT Scientific Co., Torrington, CT) to determine the antihypertensive effect of turmeric. Each rat's blood pressure was measured three times in a row, and the mean value was determined. In Wistar rats, the average SBP and DBP ranges are $103 \pm 1.1 \mathrm{mmHg}$ and $70 \pm 1.5 \mathrm{mmHg}$, respectively.

\section{HR measurement}

An electrocardiogram (ECG) previously developed and published was used to measure the HR frequency (Hasimun et al., 2019). The ECG pattern was analyzed to obtain the distance between the R-R intervals. A short R-R interval suggested a rise in the HR.

\section{Arterial stiffness assessment}

According to previous studies, calculating the PWV was used to assess arterial stiffness (Zakaria and Hasimun, 2017). It was conducted using an ECG and a photoplethysmogram (PPG) sensor. A high index of PWV suggests higher arterial stiffness, which contributes to an increase in the inflexibility of the arterial walls. This higher stiffness happens because the energy from each blood pressure pulse is not stored in the flexible walls of the vessel.

\section{Frontal QRS-T angle measurement}

The frontal plane QRS-T axis angle measurements were carried out noninvasively using the previous method (Zakaria and Hasimun, 2019). A wide QRS-T angle enhances the likelihood of cardiovascular events. The technique was designed to obtain frontal ECG, lead by combining a PPG sensor with a four-channel ECG. From those leads, the frontal QRS-T angle was calculated. The rats were placed in an airtight chamber and given $\mathrm{CO}_{2}$ gas for 1-2 minutes. Unconscious rats were then ready for ECG recording. The electrodes were mounted on the limbs, and ECG was recorded in about 10 seconds. The QRS-T angle was determined according to the method described in the previous study.

\section{NO serum level measurement}

On day 28 , serum was obtained to determine the impact of treatment on NO levels. The Griess method was used to calculate the NO level in serum (Garmana et al., 2018). Increased 
NO concentrations suggest a vasodilator effect, which results in a decrease in pressure.

Blood samples from rats were obtained and centrifuged for 15 minutes at 2,500 g. Deproteinization was carried out by adding $1 / 20$ th of a volume of $300 \mathrm{~g} / \mathrm{l}$ zinc sulfate to serum samples, resulting in a final concentration of $15 \mathrm{~g} / \mathrm{l}$. Deproteinization is needed to avoid interference in spectrophotometer readings. NO was measured in serum that had been deproteinized by centrifugation at room temperature using the Griess assay.

\section{Data analysis}

The collected data were statistically analyzed using Statistical Package for the Social Sciences version 18 software. In summary, the difference in treatment groups revealed that the effects of the test drugs differed significantly from those of the control group $(p<0.05)$.

\section{RESULTS}

In this study, the administration of an HFHF diet was associated with a significant rise in SBP and DBP compared with the normal control group (Fig. 1). Also, the induction group had the lowest levels of NO. On the contrary, the extract-receiving group had higher NO levels than the induction group $(p<0.05)$.

At doses of 50,100 , and $200 \mathrm{mg} / \mathrm{kg}$, the group receiving the turmeric extract showed a substantial decrease in SBP and DBP than the positive control group $(p<0.05)$. The reduction was similar to that seen in the captopril group. Extracts of 50, 100, and $200 \mathrm{mg} /$ $\mathrm{kg}$ reduced SBP by $37 \%, 38 \%$, and 39\%, respectively. Meanwhile, DBP had 50 percent, 50 percent, and 51 percent, respectively.

An HFHF diet increases arterial stiffness (high PWV index) significantly $(p<0.05)$ in all groups except the normal control group (Fig. 1). Meanwhile, the group receiving the turmeric extract showed a significant reduction in arterial stiffness compared to the control group ( $p<0.05)$. Furthermore, on day 28 , the extract group's frontal QRS-T angle assessment results were substantially different from those in the positive control group (Fig. 1). The results were comparable to those seen in the captopril group. The turmeric extract was responsible for reducing hemodynamic parameters, including PWV, frontal QRS-T angle, and HR. Interestingly, it is associated with higher levels of serum NO.

\section{DISCUSSION}

The following are the main findings of our research: (1) in Wistar rats, an HFHF diet caused hypertension, as shown by high SBP and DBP. (2) Cardiovascular function was compromised by the HFHF diet, which was linked to arterial stiffness and widening of the frontal QRS-T angle of the heart. (3) A prolonged diet decreases vasodilation and exacerbates hypertension by reducing NO levels. (4) The turmeric extract lowered blood pressure by increasing arterial compliance, reducing the wide frontal QRS-T angle, and maintaining NO vasodilation.

It has been proven that an HFHF diet in rats causes hypertension associated with NO deficiency. This study analyzed the effects of turmeric extract on hypertension related to chronic insufficiency NO. As presented in Figure 1, we observed a significant increase in SBP and DBP after administering an HFHF diet for 28 days. However, turmeric extract supplementation and treatment with positive control drugs (captopril) led to a significant decrease in SBP and DBP in hypertensive rats (Fig. 1). This result agrees with previously described studies where an HFHF diet is an efficient animal model and clearly describes the components of metabolic syndrome, including significant hypertension, obesity, insulin resistance, dyslipidemia, and hyperuricemia (Zhang et al.,

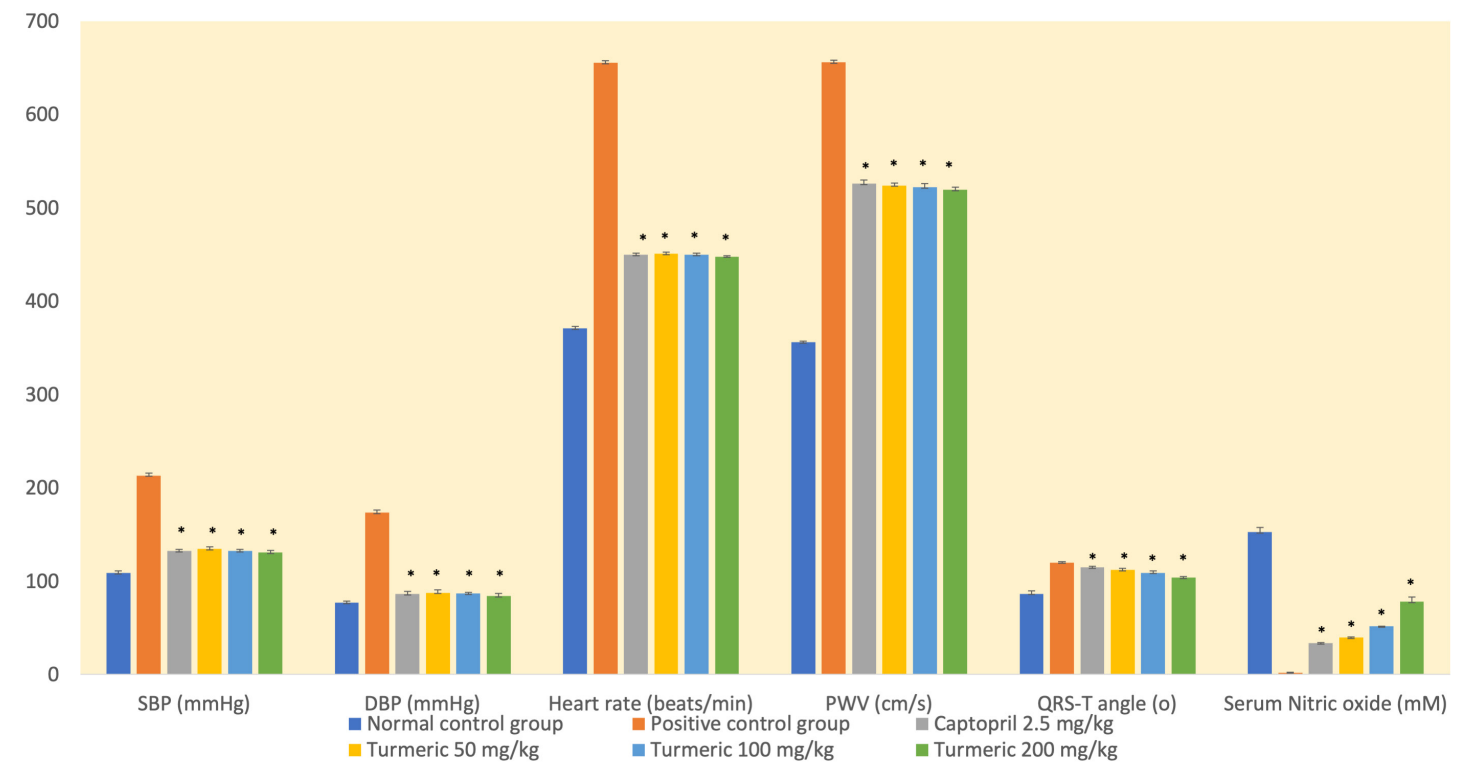

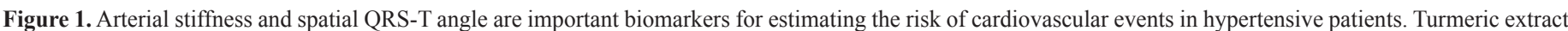

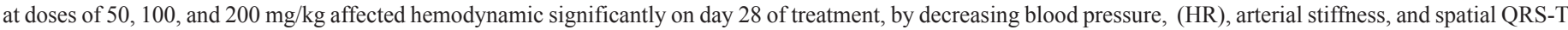

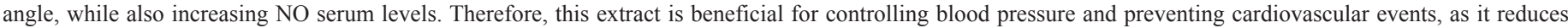

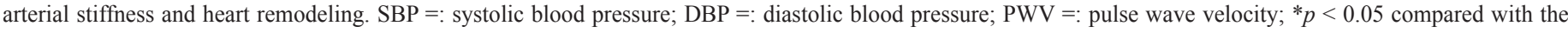
positive control group. 
2015). This diet induces hypertension by lowering the expression and function of the calcium channel, which plays a vital role in regulating arterial resistance, thus controlling blood pressure (Gradel et al., 2018).

Moreover, high-fat diets cause increases in mean arterial pressure (MAP), HR, and visceral lipid deposits, whereas fructose diets cause lipid accumulation in the liver and kidneys (de Castro et al., 2013). In a hypertensive state, an HFHF diet causes left ventricular hypertrophy (Sharma et al., 2007), which is positively associated with the widening frontal QRS-T angle (Cortez et al., 2017).

Administration of an HFHF diet increased arterial stiffness in hypertensive rats (Fig. 1). However, treatment with turmeric extract lowers arterial stiffness, supported by a significant decrease in PWV values. These results are in line with previous studies describing that the increase in arterial stiffness occurs due to increased oxidative stress, which plays a significant role in developing endothelial dysfunction (Kaprinay et al., 2017). In turn, this diet activates the renin-angiotensin-aldosterone systems and the sympathetic nervous system in the kidneys, which has been reported to cause a rise in blood pressure (Komnenov et al., 2019). Therefore, arterial stiffness assessment is an important biomarker factor for predicting cardiovascular risk in hypertensive patients (Laurent et al., 2012). Besides, arterial stiffness is also a predictor of the efficacy of hypertension management (Wang et al., 2008).

Also, turmeric extract reduced the MAP by over $40 \%$ compared to the control group. Therefore, this extract has been shown to enhance blood perfusion associated with reduced blood pressure and arterial stiffness. It can be explained that MAP, which refers to the continuous state of blood pressure, significantly affects cardiac output and peripheral resistance. It represents the cardiovascular system's physiological state that adequate arterial pressure regulates blood perfusion to all vital organs. Therefore, the increase in MAP is closely related to arterial stiffness (Tanaka et al., 2016).

An HFHF diet widened the QRS-T angle in hypertensive rats (Fig. 1). However, treatment with turmeric extract reduced a wide QRS-T angle. The frontal plane QRS-T angle, defined as the spatial angle between ventricular depolarization and repolarization, is another useful cardiovascular biomarker. The QRS-T angle could predict the risk of developing heart failure in hypertension patients (Raposeiras-Roubín et al., 2014), the incidence of coronary heart disease (Rautaharju et al., 2006), or even the potential risk of heart failure in men and women who were considered to be cardiovascular risk-free (Rautaharju et al., 2007).

Arterial stiffness and the QRS-T angle are closely linked to the concentration of NO. Furthermore, NO is an endothelial antiatherogenic molecule that plays a role in regulating vascular tone. In turn, their plasma concentration correlates to endothelial function, while a reduction indicates endothelial dysfunction (Wilkinson et al., 2004). The decrease in NO bioavailability is accompanied by increased blood pressure, contributing to increased arterial stiffness (Hermann et al., 2006). Therefore, any class of drugs that could increase the amount of NO may reduce arterial stiffness, leading to reduced blood pressure (Van Bortel et al., 2001). In hypertensive rats, turmeric extract supplementation increased serum NO levels, accompanied by decreased SBP and DBP, arterial stiffness, and the QRS-T angle (Fig. 1). These results are in line with previous research that curcumin as a turmeric bioactive compound increases NO levels by activating the expression of endothelial nitric oxide synthase (eNOS), thereby reducing arterial stiffness (Nakmareong et al., 2012).

Furthermore, this research discovered that high blood pressure is associated with a rise in HR. Turmeric extract substantially decreased the HR of the animal models (Fig. 1). This outcome supports curcumin research, in which the doxorubicininduced animal model has a cardioprotective effect (Jafarinezhad et al., 2019). According to previous research, increased HR is accompanied by increased blood pressure and the onset of hypertension. Therefore, this suggests that HR is linked to an increased risk of cardiovascular morbidity and mortality (Barison et al., 2011).

The overall result of this study revealed that the antihypertensive effect of the turmeric extract had been shown to affect the cardiovascular biomarkers, such as arterial stiffness and the QRS-T angle, linked to an increase in NO levels. Further research remains to be carried out to determine its effect on both inducible and endothelial of nitric oxide synthase (iNOS and eNOS) expression. Also, the antihypertensive effect of turmeric extract has an impact on a decreased HR.

\section{CONCLUSION}

In conclusion, the treatment of hypertensive animals with turmeric extract resulted in remarkable improvement in all of the hypertension-induced abnormalities studied, possibly due to reduced arterial stiffness, frontal QRS-T angle shift, and increased NO levels. These findings indicate that turmeric has an essential role in modulating vascular tone. Since this work is carried out by generating NO as a vasodilator, it reduces arterial stiffness and improves endothelial function.

\section{CONFLICT OF INTEREST}

The authors report no financial or any other conflicts of interest in this work

\section{FUNDING}

Basic Research Grants from The Ministry of Research and Technology of the Republic of Indonesia.

\section{AUTHOR CONTRIBUTIONS}

All authors made substantial contributions to conception and design, acquisition of data, or analysis and interpretation of data; took part in drafting the article or revising it critically for important intellectual content; agreed to submit to the current journal; gave final approval of the version to be published; and agree to be accountable for all aspects of the work. All the authors are eligible to be an author as per the international committee of medical journal editors (ICMJE) requirements/guidelines.

\section{ETHICAL APPROVALS}

This research protocol followed ethical requirements and was approved by the Ethics and Research Committee, Faculty of Medicine, Padjadjaran University, based on letter number 640/ UN6-KEP/EC/2019. 


\section{PUBLISHER'S NOTE}

This journal remains neutral with regard to jurisdictional claims in published institutional affiliation.

\section{REFERENCES}

Akinyemi AJ, Gustavo RT, Vera MM, Nathieli BB, Jucimara B, Lizielle SO, Jeferson FG, Adriane BK, Thiago D, Marta D. Effect of ginger and turmeric rhizomes on inflammatory cytokines levels and enzyme activities of cholinergic and purinergic systems in hypertensive rats. Planta Med, 2016; 82(07):612-20.

Barison A, Giuseppe V, Luigi EP, Lorenzo G, Michele E, Claudio P. Markers of arrhythmogenic risk in hypertensive subjects. Curr Pharm Des, 2011; 17(28):3062-73.

Cortez D, Schlegel TT, Ackerman MJ, Bos JM. ECGderived spatial QRS-T angle is strongly associated with hypertrophic cardiomyopathy. J Electrocardiol, 2017; 50(2):195-202.

de Castro UGM, Marcelo ES, Wanderson GDL, Maria JCS, Andréia CA. Age-dependent effect of high-fructose and high-fat diets on lipid metabolism and lipid accumulation in liver and kidney of rats. Lipids Health Dis, 2013; 12(1):1-11.

Garmana AN, Elin YS, Irda F. Antihypertension study of Anredera cordifolia (Ten). V. steenis extract and its fractions in rats through dexamethasone induction and nitric oxide release. Asian J Pharm Clin Res, 2018; $1: 278-82$.

Gradel AKJ, Max S, Charlotte MS, Niels HHR, Lars JJ. Longterm diet-induced hypertension in rats is associated with reduced expression and function of small artery SKCa, IKCa, and Kir2. 1 Channel. Clin Sci, 2018; 132(4):461-74.

Hasimun P, Yani M, Agus S, Dewa AES. Prevention of hypertension and arterial stiffness by combination of centella asiatica and Curcuma longa in rats. Asian J Biol Sci, 2019; 12(2):173-79.

Hermann M, Andreas F, Thomas FL. Nitric oxide in hypertension. J Clin Hypertens (Greenwich), 2006; 8(12 Suppl 4):17-29.

Jafarinezhad Z, Rafati A, Ketabchi F, Noorafshan A, KarbalayDoust S. Cardioprotective effects of curcumin and carvacrol in doxorubicintreated rats: stereological study. Food Sci Nutr, 2019; 7(11):3581-88.

Kaprinay B, Gáspárová Z, Lipták B, Frimmel K, Sotníková R. Endothelial dysfunction in experimental models of metabolic syndromeeffect of fructose. Eur Pharm J, 2017; 64(1):4-6.

Komnenov D, Peter EL, Noreen FR. Hypertension associated with fructose and high salt: renal and sympathetic mechanisms. Nutrients, $2019 ; 11(3): 569$

Laurent S, Maureen A, Hélène B, Pierre B. Aortic stiffness as a tissue biomarker for predicting future cardiovascular events in asymptomatic hypertensive subjects. Ann Med, 2012; 44(Sup1):S93-97. 2014; 64(1):13-8.

Mitchell GF. Arterial stiffness and hypertension. Hypertension,

Nakmareong S, Upa K, Poungrat P, Veerapol K, Bunkerd K, Wanida D, Parichat P, Chada P. Tetrahydrocurcumin alleviates hypertension, aortic stiffening and oxidative stress in rats with nitric oxide deficiency. Hypertens Res, 2012; 35(4):418-25.

Niiranen TJ, Bindu K, Naomi MH, Emelia JB, Gary FM, Ramachandran SV. Relative contributions of arterial stiffness and hypertension to cardiovascular disease: the framingham heart study. J Am Heart Assoc, 2016; 5(11):e004271.

Oehler A, Trevor F, Charles AH, Larisa GT. QRS-T angle: a review. Ann Noninvasive Electrocardiol, 2014; 19(6):534-42.

Raposeiras-Roubín RS, Alejandro VL, Noelia BC, Andrea LL, María CB, Rita FG, Alberto GC, Moises RM, Jose MGA, Emad AA, Jose
RGJ. Usefulness of the QRS-T angle to improve long-term risk stratification of patients with acute myocardial infarction and depressed left ventricular ejection fraction. Am J Cardiol, 2014; 113(8):1312-19.

Rautaharju PM, Charles K, Joseph CL, Andrea LC. Electrocardiographic abnormalities that predict coronary heart disease events and mortality in postmenopausal women: the women's health initiative. Circulation, 2006; 113(4):473-80.

Rautaharju PM, Ron JP, Joy W, Zhu MZ, Richard C, Gerardo $\mathrm{H}$. Electrocardiographic predictors of new-onset heart failure in men and in women free of coronary heart disease (from the atherosclerosis in communities [ARIC] study). Am J Cardiol, 2007; 100(9):1437-41.

Selvaraj S, Leonard I, Michael AB, Benjamin HF, Roberto ML, Eva EM, Sanjiv JS. Association of the frontal QRS-T angle with adverse cardiac remodeling, impaired left and right ventricular function, and worse outcomes in heart failure with preserved ejection fraction. J Am Soc Echocardiogr, 2014; 27(1):74-82.

Sharma N, Isidore CO, Monika KD, Janean J, Celvie LY, Margaret PC, Paul E, Brian DH, William CS. High fructose diet increases mortality in hypertensive rats compared to a complex carbohydrate or high fat diet. Am J Hypertens, 2007; 20(4):403-9.

Sukandar EY, Permana H, Adnyana IK, Sigit JI, Ilyas RA, Hasimun P, Mardiyah D. Clinical study of turmeric (Curcuma longa L.) and garlic (Allium sativum L.) extracts as antihyperglycemic and antihyperlipidemic agent in type- 2 diabetes-dyslipidemia patients. Int $J$ Pharmacol, 2010; 6(4):456-63.

Sun Z. Aging, arterial stiffness, and hypertension. Hypertension, $2015 ; 65(2): 252-56$.

Tanaka H, Heiss G, McCabe EL, Meyer ML, Shah AM, Mangion JR, Wu J, Solomon SD, Cheng S. Hemodynamic correlates of blood pressure in older adults: the Atherosclerosis risk in communities (ARIC) study. J Clin Hypertens, 2016; 18(12):1222-27.

Van Bortel LM, Struijker-Boudier HA, Safar ME. Pulse pressure, arterial stiffness, and drug treatment of hypertension. Hypertension, 2001; 38(4):914-21.

Wang X, James CKJ, Allan DS, Giora ZF. Assessment of arterial stiffness, a translational medicine biomarker system for evaluation of vascular risk. Cardiovasc Ther, 2008; 26(3):214-23.

Wilkinson IB, Stanley SF, John RC. Nitric oxide and the regulation of large artery stiffness: From physiology to pharmacology. Hypertension, 2004; 44 2):112-6.

Zakaria H, Hasimun P. Non-invasive pulse wave velocity measurement in mice. International Seminar on Sensors, Instrumentation, Measurement and Metrology (ISSIMM), Malang, Indonesia, pp 95-98, 2017.

Zakaria H, Patonah H. Frontal QRS-T angle measurement in mice. International Symposium on Electronics and Smart Devices (ISESD), IEEE, pp 1-4, 2019.

Zhang Z, Zhiquan W, Zhen Y, Yixin N, Weiwei Z, Xiaoyong LI, Yueming LIU, Hongmei Z, Li Q, Qing SU. A novel mice model of metabolic syndrome: the high-fat-high-fructose diet-fed ICR mice. Exp Anim, 2015; 64(4):435-42.

How to cite this article:

Hasimun P, Sulaeman A, Hidayatullah A, Mulyani Y. Effect of Curcuma longa L. extract on noninvasive cardiovascular biomarkers in hypertension animal models. J Appl Pharm Sci, 2021; 11(08):085-089. 